\title{
COVID-19: Vaccine and antimicrobial resistance what are the global implications?
}

\author{
Jared Robinson, Alexandra Leclézio, Indrajit Banerjee
}

Sir Seewoosagur Ramgoolam Medical College, Belle Rive, Mauritius

Keywords: BNT162 vaccine, COVID-19 pandemic, COVID-19 Vaccines, Drug Resistance, mRNA-1273 vaccine, P.1 SARSCoV-2 variant, SARS-CoV-2 B.1.351 variant

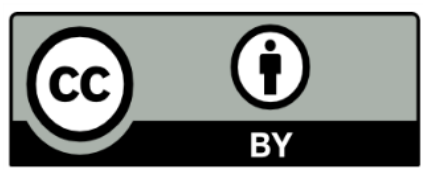

This work is licensed under a Creative Commons Attribution 4.0 Unported License.
Abstract

Antimicrobial resistance is a worldwide and highly quantified risk to global health and is more prevalent than resistance developed in vaccines as both antimicrobial resistance and vaccine resistance develop in different settings and because of alternate mechanisms. Vaccines act as a preventative measure and allow the immune system to kill any pathogen in the initial phases when the load is relatively low. This circumvents the replication of the pathogen and thus prevents the formation of mutations and furthermore resistance which is attributed to those mutations. Mutations in the target and or binding sites of a said therapeutic regime confer resistance more often in antimicrobials than they do vaccines.

The alteration of a vaccines binding site does not confer resistance as a vaccine produces a wide spectrum of antibodies due to multiple epitopes on the said antigen, the remaining antibodies are thus still protective. In antimicrobials however, the drug targets a specific site and is not dynamic and thus if a mutation of the site arises, the drug efficacy is reduced.

Although vaccine resistance is less quantified it may also pose a substantial risk to Global health as currently evident with the COVID-19 pandemic. The current global pandemic caused by SARS-CoV-2 has developed a host of mutations which are displaying a degree of resistance and reduced efficacy to the vaccines. This reduced efficacy and resistance of the mutations to current vaccination programmes, poses a risk to global health. It is vital for new vaccines to be synthesized to specifically be active against the variants. It is likely that the synthesis and development of new vaccines to counter new variants as they arise will be an ongoing process. It is evident in future that new vaccines to the mutations in COVID-19 may have to be developed as they are for the seasonal influenzae virus.

${ }^{*}$ Corresponding Author:

Dr. Indrajit Banerjee, MBBS, MD

Associate Professor

Department of Pharmacology

Sir Seewoosagur Ramgoolam Medical College, Belle Rive, Mauritius +230-58832236

indrajit18@gmail.com

Orcid id: https://orcid.org/0000-0003-2880-4695 
Uses of antimicrobials vs vaccines:

Antimicrobials and vaccines are used in different settings for different purposes. Vaccines are generally synthesized for the use against viruses except the MCV4, tetanus toxoid vaccine, Tdapdiphtheria toxoid and acellular pertussis pneumococcal, and Hib which are bacterial vaccines. Whereas antimicrobials are generally used against bacteria. ${ }^{3}$

\section{Resistance in vaccines:}

The emergence of resistance towards vaccines is a far less quantified risk to global health. Vaccines act as a preventative measure and allow the immune system to kill any pathogen in the initial phases when the load is relatively low. This circumvents the replication of the pathogen and thus prevents the formation of mutations and furthermore resistance which is attributed to those mutations. Vaccines develop resistance so seldomly that the same formulas of vaccines have been used for decades, without the development of resistance. Although less quantified resistance to vaccines however does develop, for example resistance of the influenza virus. ${ }^{4-8}$

The influenza virus undergoes mutations at a high rate. The periodic antigenic drift and shift of the virus is the culprit. Antigenic drift occurs due to minimally quantified mutations and alterations and is the overriding factor for a new vaccine to be synthesized each year. Antigenic shift however occurs due to a major alteration and mutation and thus leads to an influenza epidemic.

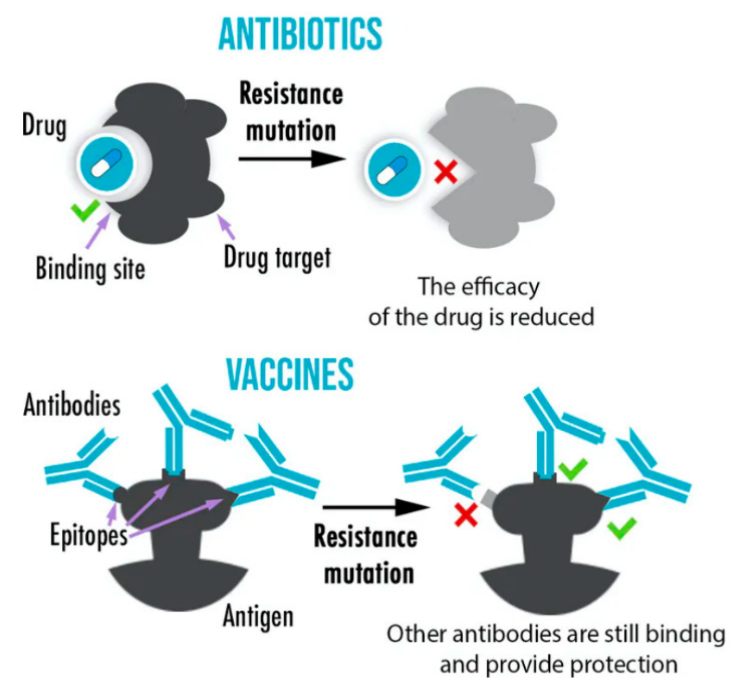

Figure 1: Target site of binding for antibiotics and vaccines ${ }^{8}$

Figure 1 below depicts how various mutations in the target and or binding sites of a said therapeutic regime confer resistance more often in antimicrobials than they do vaccines. The alteration of a vaccines binding site does not confer resistance as a vaccine produces a wide spectrum of antibodies due to multiple epitopes on the said antigen, the remaining antibodies are thus still protective. In antimicrobials however, the drug targets a specific site and is not dynamic and thus if a mutation of the site arises, the drug efficacy is reduced.
SARS-CoV-2 variants and vaccination:

The current global pandemic caused by SARS-CoV-2 has developed a host of mutations which are displaying a degree of resistance and reduced efficacy to the vaccines. A study conducted on 20 subjects who had received either the Moderna (mRNA-1273) or Pfizer-BioNTech (BNT162b2) vaccine showed very good efficacy to infections with the wild type of virus. The efficacy on the mutated P.1 Brazilian variant and the B.1.351 South African strains however was markedly reduced. The wild type and mutated strains share a host of common target epitopes and receptor binding domains which should theoretically confer a level immunity. In actuality this does not hold true as out of the 17 most vital monoclonal antibodies produced 14 of which have a diminished and or annulled neutralization capacity in the mutated strains. This reduced efficacy and resistance of the mutations to current vaccination programmes, pose a risk to global health. It is vital for new vaccines to be synthesized to specifically be active against the variants. ${ }^{9-11}$

Resistance in antimicrobials:

Currently bacteria have developed resistance to every antibiotic on the market. Resistance develops in a host of manners namely through mutations, transduction, transformation, lysogenic conversion, transposons and conjugation. The overprescribing and overuse of antimicrobial agents has further provided the fertile soil for the emergence of resistant strains as it applies a local pressure for resistance to occur via the culling of "good" normal gut flora and fauna. ${ }^{4,5}$

The improper use of antibiotics by patients via the premature cessation of the prescribed course and via the failure to complete the course has caused the development of MDR-TB (multidrug resistant tuberculosis). ${ }^{6}$

The resistance of bacteria to antimicrobial agents is further enhanced by the "discovery void". No new antimicrobial agents have been discovered since 1987 and thus means that the antibiotics in use today have been in circulation for an extended period of time. Time and re-use as well as cross resistance forms a hotbed for the emergence of antimicrobial resistance. ${ }^{7}$

Vaccine resistance vs antimicrobial resistance:

The mechanisms by which these two vastly different therapeutic regimes act is cause for antimicrobials to be far more at risk to developing resistance as opposed to vaccines. Antimicrobials are used in situations where a colony of bacteria is well established and the bacterial load is in the high millions. The bacteria divide at an exponential rate, the more divisions that occur equates to a greater likelihood of errors in DNA and RNA replication. These errors translate into a higher rate of mutations. ${ }^{12,13}$

Vaccines however act by a completely different mechanism. Vaccines are preventative in nature and thus stimulate the hosts immune system to produce antibodies long before the wild type of pathogen is actually exposed to the host. This ensures that when the pathogen is exposed to a host that it is rapidly eradicated when the load is still minimal, as opposed to antimicrobials which face a very high bacterial load. This destruction and neuralization of the pathogen at such an early phase ensure that replication is kept to a minimum, and thus reduces the likelihood of mutations occurring. ${ }^{14}$

An advantage that vaccines have over antimicrobial agents is the fact that the human immune system is highly complex and 
advanced. It does not only produce a single antibody to a specific antigen, but produces a wide range of different antibodies to various epitopes of the antigen with which the patient was immunized. This confers a wider range of activity as opposed to antimicrobial agents which will act on a single specific enzyme or target.

The new strains of SARS-CoV-2 however are cause for great concern as their resistance to vaccine monoclonal antibody neutralization equates to infections and therefore jeopardizes the entire global vaccination campaign. It is therefore pertinent for new specific vaccines to be synthesized which are active against the variants. It is likely that the synthesis and development of new vaccines to counter new variants as they arise will be an ongoing process. It is evident in future that new vaccines to the mutations in COVID-19 may have to be developed as they are for the seasonal influenzae virus. $8,9,10,15$

\section{Conclusion:}

Antimicrobial resistance is a well-established global health concern. Vaccine resistance is of a far less quantitated concern; however, the SARS-CoV-2 pandemic has raised great concerns about vaccine efficacy and resistance due to the development of the new SARS-CoV-2 variants. It is likely that the synthesis and development of new vaccines to counter new variants as they arise will be an ongoing process in future. Rational, judicious prescribing and dispensing of drugs as well as better patient compliance and adherence to rules and regulations to curb the spread of the virus should be used at the forefront to safeguard and prevent the development of further resistance.

References:

1. D'Costa VM, King CE, Kalan L, Morar M, Sung WW, Schwarz C, et al. Antibiotic resistance is ancient. Nature. 2011 Sep;477(7365):45761.

https://doi.org/10.1038/nature10388

2. Brown D. Antibiotic resistance breakers: can repurposed drugs fill the antibiotic discovery void?. Nature reviews Drug discovery. 2015 Dec;14(12):821-32.

https://doi.org/10.1038/nrd4675

3. Mendoza N, Ravanfar P, Satyaprakah A, Pillai S, Creed R. Existing antibacterial vaccines. Dermatologic Therapy. 2009; 22: 129-142.

https://doi.org/10.1111/j.1529-8019.2009.01225.x

4. Khachatourians GG. Agricultural use of antibiotics and the evolution and transfer of antibiotic-resistant bacteria. CMAJ. 1998 Nov 3;159(9):1129-36.

5. Rao GG. Risk factors for the spread of antibiotic-resistant bacteria. Drugs. 1998 Mar 1;55(3):323-30.

https://doi.org/10.2165/00003495-199855030-00001

6. World Health Organization - WHO. Antibacterial agents in clinical development: an analysis of the antibacterial clinical development pipeline, including tuberculosis [Internet]. Geneva; 2017 WHO/EMP/IAU/2017.). [cited 2021 March 4]. Available from: http://www.who.int/medicines/areas/rational_use/antibacterial_ agents_clinical_development/en/

7. Guarnaccia T, Carolan LA, Maurer-Stroh S, Lee RT, Job E, Reading PC, et al. Antigenic drift of the pandemic 2009 A(H1N1) influenza virus in A ferret model. PLoS Pathog. 2013;9(5):e1003354.

https://doi:10.1371/journal.ppat.1003354

8. Why resistance is common in antibiotics, but rare in vaccines. The Conversation. [Internet]. [cited 2021 March 24]. Available from: https://theconversation. com/why-resistance-is-common-in-antibiotics-butrare-in-vaccines -152647 ? utm_medium =Social $\&$ utm source $=$ Twitter\&fbclid=IwAR1 IQDkK1Dg0XCv2YCXGdpox7q pQXI-FPeUWVOIQIBZXIoW-zmXONtCWZO\#Echobox=1610437207

9. Wang Z, Schmidt F, Weisblum Y, Muecksch F, Barnes CO, Finkin $S$, et al. mRNA vaccine-elicited antibodies to SARS-CoV-2 and circulating variants. Nature. 2021 Feb 10.

https://doi.org/10.1038/s41586-021-03324-6

10. Mohabeer P, Banerjee I, Kashyap A, Shukla A, Robinson J, Ramdhan ND. SARS-CoV-2-an update in virology: A Narrative Review. Journal of Biomedical Sciences. 2020 Dec 31;7(2):77-87.

https://doi.org/10.3126/jbs.v7i2.34007

11. Fontanet A, Autran B, Lina B, Kieny MP, Karim SS, Sridhar D. SARS-CoV-2 variants and ending the COVID-19 pandemic. The Lancet. 2021 Feb 11.

https://doi.org/10.1016/S0140-6736(21)00370-6

12. Aiken AM, Allegranzi B, Scott JA, Mehtar S, Pittet D, Grundmann $\mathrm{H}$. Antibiotic resistance needs global solutions. The Lancet infectious diseases. $2014 \mathrm{Jul}$ 1;14(7):550-1

https://doi.org/10.1016/S1473-3099(14)70709-1

13. Zhou J, Dong Y, Zhao X, Lee S, Amin A, Ramaswamy S, et al. Selection of antibiotic-resistant bacterial mutants: allelic diversity among fluoroquinolone-resistant mutations. The Journal of infectious diseases. 2000 Aug 1;182(2):517-25.

https://doi.org/10.1086/315708

14. Cui Z, Han SJ, Vangasseri DP, Huang L. Immunostimulation mechanism of LPD nanoparticle as a vaccine carrier. Molecular pharmaceutics. $2005 \mathrm{Feb}$ 7;2(1):22-8.

https://doi.org/10.1021/mp049907k

15. Davtyan H, Ghochikyan A, Petrushina I, Hovakimyan A, Davtyan A, Poghosyan A, et al. Immunogenicity, efficacy, safety, and mechanism of action of epitope vaccine (Lu AF20513) for Alzheimer's disease: prelude to a clinical trial. Journal of Neuroscience. 2013 Mar 13;33(11):4923-34.

https://doi.org/10.1523/JNEUROSCI.4672-12.2013 